Internat. J. Math. \& Math. Sci.

Vol. 22, No. 4 (1999) 847-854

S 0161-1712<99>22847-5

(C) Electronic Publishing House

\title{
NONLINEAR FUNCTIONAL INTEGRODIFFERENTIAL EQUATIONS IN HILBERT SPACE
}

\author{
J. Y. PARK, S. Y. LEE, and M. J. LEE
}

(Received 7 November 1996 and in revised form 3 November 1997)

\begin{abstract}
Let $X$ be a Hilbert space and let $\Omega \subset R^{n}$ be a bounded domain with smooth boundary $\partial \Omega$. We establish the existence and norm estimation of solutions for the parabolic partial functional integro-differential equation in $X$ by using the fundamental solution.
\end{abstract}

Keywords and phrases. Functional integro-differential equation, elliptic differential operators, fundamental solution, Gårding's inequality, successive approximation, norm estimation.

1991 Mathematics Subject Classification. 35A05, 35J60, 45K05.

1. Introduction. Let $X$ be a Hilbert space and let $\Omega \subset R^{n}$ be a bounded domain with smooth boundary $\partial \Omega$. We consider the following parabolic partial functional integrodifferential equation.

$$
\begin{aligned}
\frac{\partial u}{\partial t}= & \mathscr{A}_{0} u(t, x)+\mathscr{A}_{1} u(t-h, x)+\int_{-h}^{0} a(s) \mathscr{A}_{2} u(t+s, x) d s \\
& +\int_{0}^{t}\{k(t, s) G(s, u(s-h), x)+H(t, s, u(s-h, x))\} d s \\
& +F(t, u(t-h, x))+f(t, x), \quad 0<t \leq T, x \in \Omega,
\end{aligned}
$$

where $\mathscr{A}_{i}(i=0,1,2)$ are elliptic differential operators, $f$ is a forcing function, $h>0$ is a delay time, $a(s)$ is a real scalar function on $[-h, 0], G, H$, and $F$ are nonlinear functions, and $k$ is a kernel. The boundary condition attached to (1.1) is, e.g., given by the Dirichlet boundary condition

$$
\left.u\right|_{\partial \Omega}=0, \quad 0<t \leq T,
$$

and the initial condition is given by

$$
u(\theta, x)=g(\theta, x), \quad \theta \in[-h, 0], x \in \Omega .
$$

From [4], the above mixed problems (1.1), (1.2), and (1.3) can be formulated abstractly as

$$
\begin{gathered}
\frac{d u(t)}{d t}=A_{0} u(t)+A_{1} u(t-h)+\int_{-h}^{0} a(s) A_{2} u(t+s) d s \\
+\int_{0}^{t}\left\{k(t, s) G\left(s, u_{s}\right)+H\left(t, s, u_{s}\right)\right\} d s \\
+F\left(t, u_{t}\right)+f(t), \quad 0<t \leq T, \\
u(\theta)=g(\theta), \quad \theta \in[-h, 0],
\end{gathered}
$$


where the state $u(x)$ of the system (1.5) lies in an appropriate Hilbert space and $A_{i}(i=0,1,2)$ are unbounded operators associated with $\mathscr{A}_{i}(i=0,1,2)$, respectively. Next, we explain the notation $u_{t}$ in (1.5). Let $I=[-h, 0]$. If a function $u(t)$ is continuous from $I \cup[0, T]$ into a Hilbert space $X$, then $u_{t}$ is an element in $C=C([-h, 0] ; X)$, which has the point-wise definition

$$
u_{t}(\theta)=u(t+\theta) \text { for } \theta \in I
$$

Let $\Delta_{T}=\{(s, t) ; 0 \leq s \leq t \leq T\}$. We assume in (1.5) that $G:[0, T] \times C \rightarrow X, H: \Delta_{T} \times C \rightarrow$ $X, F:[0, T] \times C \rightarrow X$ and the kernel $k: \Delta_{T} \rightarrow R$ ( $R$ denotes the set of real numbers) are continuous, $f:[0, T] \rightarrow V^{*}$ with some enlarged space $V^{*} \supset H$ and $g:[-h, 0] \rightarrow V$ with some dense subspace $V \subset H$. It is assumed that the inclusions $V \subset H \subset V^{*}$ are continuous and $V^{*}$ is the dual space of $V$.

Many authors $[2,8]$ studied the following delay differential equation:

$$
\begin{gathered}
\frac{d u(t)}{d t}=A_{0} u(t)+A_{1} u(t-h)+\int_{-h}^{0} a(s) A_{2} u(t+s) d s+f(t), \quad \text { a.e. } t \geq 0, \\
u(\theta)=g(\theta), \quad \theta \in[-h, 0] .
\end{gathered}
$$

The fundamental solution is constructed in Tanabe [8]. In this paper, we establish the existence and norm estimation of solutions for the equation (1.5) by using the fundamental solution.

2. Preliminaries. Let $H$ be a pivot complex Hilbert space and $V$ be a complex Hilbert space such that $V$ is dense in $H$ and the inclusion map $i: V \rightarrow H$ is continuous. The norms of $H, V$, and the inner product of $H$ are denoted by $|\cdot|,\|\cdot\|$, and $\langle\cdot, \cdot\rangle$, respectively. Identifying the antidual of $H$ with $H$, we may consider that $V \subset H \subset V^{*}$. The norm of the dual space $V^{*}$ is denoted by $\|\cdot\|_{*}$. We consider the following linear functional differential equation on the Hilbert space $H$.

$$
\begin{gathered}
\frac{d u(t)}{d t}=A_{0} u(t)+A_{1} u(t-h)+\int_{-h}^{0} a(s) A_{2} u(t+s) d s+f(t), \quad \text { a.e. } t \geq 0, \\
u(0)=g^{0}, \quad u(s)=g^{1}(s), \quad \text { a.e. } s \in[-h, 0] .
\end{gathered}
$$

Let $a(u, v)$ be a bounded sesquilinear form defined in $V \times V$ satisfying Gårding's inequality

$$
\operatorname{Re} a(u, u) \geq c_{0}\|u\|^{2}-c_{1}|u|^{2},
$$

where $c_{0}>0$ and $c_{1} \geq 0$ are real constants. Let $A_{0}$ be the operator associated with this sesquilinear form

$$
\left\langle v, A_{0} u\right\rangle=-a(u, v), \quad u, v \in V,
$$

where $\langle\cdot, \cdot\rangle$ denotes the duality pairing between $V$ and $V^{*}$. The operator $A_{0}$ is bounded linear from $V$ into $V^{*}$. The realization of $A_{0}$ in $H$, which is the restriction of $A_{0}$ to the domain $D\left(A_{0}\right)=\left\{u \in V: A_{0} u \in H\right\}$, is also denoted by $A_{0}$. It is proved in Tanabe [6] that $A_{0}$ generates an analytic semigroup $e^{t A_{0}}=T(t)$ both in $H$ and $V^{*}$ and that $T(t)$ : $V^{*} \rightarrow V$ for each $t>0$. Throughout this paper, it is assumed that each $A_{i}(i=1,2)$ is bounded and linear from $V$ to $V^{*}$ (i.e., $A_{i} \in \mathscr{L}\left(V, V^{*}\right)$ ) such that $A_{i}$ maps $D\left(A_{0}\right)$ 
endowed with the graph norm of $A_{0}$ to $H$ continuously. The real valued scalar function $a(s)$ is assumed to be Hölder continuous on $[-h, 0]$. We introduce a Stieltjes measure $\eta$ given by

$$
\eta(s)=-\chi_{(-\infty,-h]}(s) A_{1}-\int_{s}^{0} a(\xi) d \xi A_{2}: V \rightarrow V^{*}, \quad s \in[-h, 0],
$$

where $X_{(-\infty,-h]}$ denotes the characteristic function of $(-\infty,-h]$. Then the delay term in (2.1) is written simply as $\int_{-h}^{0} d \eta(s) u(t+s)$. The fundamental solution $W(t)$ of (2.1) is defined as a unique solution of

$$
W(t)=\left\{\begin{array}{l}
T(t)+\int_{0}^{t} T(t-s) \int_{-h}^{0} d \eta(\xi) W(\xi+s) d s, \quad t \geq 0 \\
0, \quad t<0
\end{array}\right.
$$

and $W(t)$ is constructed by Tanabe [7] under the Hölder continuity of $a(s)$.

THEOREM 2.1 [2]. The fundamental solution $W(t)$ is strongly continuous in $V, H$, and $V^{*}$, and for each $t>0, W(t): V^{*} \rightarrow V$. Furthermore, $W(t)$ satisfies

$$
\frac{d}{d t} W(t)=A_{0} W(t)+\int_{-h}^{0} d \eta(s) W(t+s), \text { a.e. } t>0 .
$$

For each $t>0$, we define the operator valued function $U_{t}(\cdot)$ by

$$
U_{t}(s)=\int_{-h}^{s} W(t-s+\xi) d \eta(\xi): V \longrightarrow V \text {, a.e. } s \in[-h, 0] \text {. }
$$

Let $T>0$ be fixed. Associated with $U_{t}(\cdot)$, we consider the operator $u: L^{2}(-h, 0 ; V) \rightarrow$ $L^{2}(0, T ; V)$ defined by

$$
\left(u g^{1}\right)(t)=\int_{-h}^{0} U_{t}(s) g^{1}(s) d s, \quad t \in[0, T]
$$

for $g^{1} \in L^{2}(-h, 0 ; V)$.

THEOREM 2.2 [8]. Let $T>0$ be fixed. Assume that $f \in L^{2}\left(0, T ; V^{*}\right)$ and $g=\left(g^{0}, g^{1}\right) \in$ $H \times L^{2}(-h, 0 ; V)$. Then there exists a unique solution $u(t)=u(t ; f, g)$ of $(2.1)$ on $[0, T]$ satisfying

$$
u \in L^{2}(0, T ; V) \cap W^{1,2}\left(0, T ; V^{*}\right) \subset C([0, T] ; H) .
$$

Further, for each $T>0$, there is a constant $K_{T}$ such that

$$
\int_{0}^{T}\|u(t)\|^{2} d t+\int_{0}^{T}\left\|\frac{d u(t)}{d t}\right\|_{*}^{2} d t \leq K_{T}\left(\left|g^{0}\right|^{2}+\int_{-h}^{0}\left\|g^{1}(s)\right\|^{2} d s+\int_{0}^{T}\|f(t)\|_{*}^{2} d t\right) .
$$

This solution $u(t)$ is represented by

$$
u(t ; f, g)=W(t) g^{0}+\left(u g^{1}\right)(t)+\int_{0}^{t} W(t-s) f(s) d s .
$$

In what follows, in order to consider the solutions in the state space $C=C([-h, 0] ; H)$, we assume that $g=\left(g^{0}, g^{1}\right)$ is continuous in $H$, i.e.,

$$
g(0)=g^{0}, \quad g(\cdot)=g^{1}(\cdot) \in C([-h, 0] ; H) .
$$


Let

$$
\hat{u}(t ; f, g)=\left\{\begin{array}{l}
u(t ; f, g), \quad t \in[0, T], \\
g(t), \quad t \in[-h, 0] .
\end{array}\right.
$$

Then, by Theorem 2.2, we get

$$
\hat{u}(\cdot ; f, g) \in C([-h, T] ; H)
$$

if (2.12) is satisfied.

3. Existence and uniqueness of functional integro-differential equations. Using the fundamental solution $W(t)$ in Section 2, we consider the following abstract functional integral equation.

$$
\begin{aligned}
v(t)= & u(t ; f, g) \\
& +\int_{0}^{t} W(t-s)\left[\int _ { 0 } ^ { s } \left\{k(s, \tau) G\left(\tau, v_{\tau}\right)\right.\right. \\
& \left.\left.+H\left(s, \tau, v_{\tau}\right)\right\} d \tau+F\left(s, v_{s}\right)\right] d s, \quad 0<t \leq T, \\
v(\theta)= & g(\theta), \quad \theta \in[-h, 0],
\end{aligned}
$$

where $u(t ; f, g)$ is given by (2.11).

We list the following hypotheses.

$\left(\mathrm{A}_{1}\right)$ The nonlinear functions $G:[0, T] \times C \rightarrow H, H: \Delta_{T} \times C \rightarrow H, F:[0, T] \times C \rightarrow H$, and the kernel $k: \Delta_{T} \rightarrow R$ ( $R$ denotes the set of real numbers) are continuous.

$\left(\mathrm{A}_{2}\right)$ Let $b_{1}, b_{3}:[0, T] \rightarrow R, b_{2}: \Delta_{T} \rightarrow R^{+}$be continuous functions such that

$$
\begin{gathered}
|G(t, \phi)-G(t, \bar{\phi})|_{X} \leq b_{1}(t)|\phi-\bar{\phi}|_{C} ; \\
|H(t, s, \phi)-H(t, s, \bar{\phi})|_{X} \leq b_{2}(t, s)|\phi-\bar{\phi}|_{C} ; \\
|F(t, \phi)-F(t, \bar{\phi})|_{X} \leq b_{3}(t)|\phi-\bar{\phi}|_{C}
\end{gathered}
$$

for $t, s \in[0, T], \phi, \bar{\phi} \in C$.

$\left(\mathrm{A}_{3}\right)$ The function $k(t, s)$ is Hölder continuous with exponent $\alpha$, i.e., there exists a positive constant $a$ such that

$$
\left|k\left(t_{1}, s_{1}\right)-k\left(t_{2}, s_{2}\right)\right| \leq a\left(\left|t_{1}-t_{2}\right|^{\alpha}+\left|s_{1}-s_{2}\right|^{\alpha}\right)
$$

for $t_{1}, t_{2}, s_{1}, s_{2} \in[0, T], 0<\alpha \leq 1$.

$\left(\mathrm{A}_{4}\right)$ For all $0 \leq s \leq t \leq T$,

$$
G(t, 0)=0, \quad H(t, s, 0)=0, \quad F(t, 0)=0 .
$$

THEOREM 3.1. Let $f \in L^{2}\left(0, T ; V^{*}\right)$ and $g=(g(0), g(\cdot)) \in H \times L^{2}(-h, 0 ; V)$ satisfy (2.12). Assume that the hypotheses $\left(A_{1}\right)-\left(A_{4}\right)$ hold. Then there exists a time $t_{1}>0$ such that the functional integral equation (3.1) admits a unique solution $v(t)$ on $\left[0, t_{1}\right]$.

Proof. We prove this theorem by using the method of successive approximations. Set $v^{0}(t)=u(t ; f, g), t \geq 0$. Let $\hat{v}^{0}(t)$ be the extension of $v^{0}(t)$ on $[-h, T]$ by (2.13). Then, by the assumptions on $f$ and $g$, we have $\hat{v}^{0}(t) \in C([-h, T] ; H)$. By hypotheses $\left(\mathrm{A}_{1}\right)-\left(\mathrm{A}_{4}\right)$, we define $\left\{\hat{v}^{n}\right\}_{n=0}^{\infty} \subset C([-h, T] ; H)$ successively by 


$$
\begin{aligned}
\hat{v}^{n}(t)= & u(t ; f, g) \\
+\int_{0}^{t} W(t-s)[ & \int_{0}^{s}\left\{k(s, \tau) G\left(\tau, \hat{v}_{\tau}^{n-1}\right)\right. \\
& \left.\left.+H\left(s, \tau, \hat{v}_{\tau}^{n-1}\right)\right\} d \tau+F\left(s, v_{s}^{n-1}\right)\right] d s, \quad 0<t \leq T, \\
& \hat{v}^{n}(\theta)=g(\theta), \quad \theta \in[-h, 0] .
\end{aligned}
$$

It is obvious that $M=\sup _{t \in[0, T]}\|W(t)\|_{L(H)}$ is finite and that

$$
\hat{v}^{n+1}(\theta)-\hat{v}^{n}(\theta)=0, \quad \theta \in[-h, 0] .
$$

For $0 \leq t \leq T$, we have, by $\left(\mathrm{A}_{1}\right)-\left(\mathrm{A}_{4}\right)$ and the strong continuity of $W(t)$ on $[0, T]$,

$$
\begin{aligned}
\mid \hat{v}^{n+1}(t)- & \hat{v}^{n}(t) \mid \\
& =\mid \int_{0}^{t} W(t-s)\left[\int_{0}^{s}\left\{k(s, \tau) G\left(\tau, \hat{v}_{\tau}^{n}\right)+H\left(s, \tau, \hat{v}_{\tau}^{n}\right)\right\} d \tau+F\left(s, \hat{v}_{s}^{n}\right)\right] d s \\
& -\int_{0}^{t} W(t-s)\left[\int_{0}^{s}\left\{k(s, \tau) G\left(\tau, \hat{v}_{\tau}^{n-1}\right)+H\left(s, \tau, \hat{v}_{\tau}^{n-1}\right)\right\} d \tau+F\left(s, \hat{v}_{s}^{n-1}\right)\right] d s \mid \\
\leq & M \int_{0}^{t}\left[\int_{0}^{s}|k(s, \tau)|\left|G\left(\tau, \hat{v}_{\tau}^{n}\right)-G\left(\tau, \hat{v}_{\tau}^{n-1}\right)\right| d \tau d s\right. \\
& \left.\quad+\int_{0}^{s}\left|H\left(s, \tau, \hat{v}_{\tau}^{n}\right)-H\left(s, \tau, \hat{v}_{\tau}^{n-1}\right)\right| d \tau\right]+M \int_{0}^{t}\left|F\left(s, \hat{v}_{s}^{n}\right)-F\left(s, \hat{v}_{s}^{n-1}\right)\right| d s \\
& \leq M \int_{0}^{t}\left[\int_{0}^{s}\left\{|k(s, \tau)|\left|b_{1}(\tau)\right|\left\|\hat{v}_{\tau}^{n}-\hat{v}_{\tau}^{n-1}\right\|+\left|b_{2}(s, \tau)\right|\left\|\hat{v}_{\tau}^{n}-\hat{v}_{\tau}^{n-1}\right\|\right\} d \tau\right] d s \\
& +M \int_{0}^{t}\left|b_{3}(s)\right|\left\|\hat{v}_{s}^{n}-\hat{v}_{s}^{n-1}\right\| d s \\
\leq & M \int_{0}^{t}\left[K L_{1}+L_{2}\right]\left\|\hat{v}_{\tau}^{n}-\hat{v}_{\tau}^{n-1}\right\| s d s+M \int_{0}^{t} L_{3}\left\|\hat{v}_{s}^{n}-\hat{v}_{s}^{n-1}\right\| d s \\
\leq & {\left[M\left(K L_{1}+L_{2}\right) \frac{1}{2} t^{2}+M L_{3} t\right]\left\|\hat{v}^{n}-\hat{v}^{n-1}\right\|_{C([-h, T] ; H)} } \\
& =\left(c_{1} t+c_{2}\right) t\left\|\hat{v}^{n}-\hat{v}^{n-1}\right\|_{C([-h, T] ; H)},
\end{aligned}
$$

where $c_{1}=(1 / 2) M\left(K L_{1}+L_{2}\right)$ and $c_{2}=M L_{3}$. We now choose a sufficiently small constant $t_{1}>0$ such that

$$
L=\left(c_{1} t_{1}+c_{2}\right) t_{1}<1 .
$$

Then by (3.6), (3.8), and (3.9), we get

$$
\begin{aligned}
\left\|\hat{v}^{n+1}-\hat{v}^{n}\right\|_{C([-h, T] ; H)} & \leq L\left\|\hat{v}^{n}-\hat{v}^{n-1}\right\|_{C([-h, T] ; H)} \\
& \ldots \ldots \ldots \\
& \leq L^{n}\left\|\hat{v}^{1}-\hat{v}^{0}\right\|_{C([-h, T] ; H)} .
\end{aligned}
$$

This implies that $\left\{\hat{v}^{n}\right\}_{n=0}^{\infty}$ converges uniformly to some $\hat{v} \in C([-h, 0] ; H)$. Therefore,

$$
\lim _{n \rightarrow \infty} \sup _{t \in\left[0, t_{1}\right]}\left\|\hat{v}_{t}^{n}-\hat{v}_{t}\right\|_{C([-h, 0] ; H)}=0 .
$$


Hence, by letting $n \rightarrow \infty$ in (3.5), in view of $\left(\mathrm{A}_{1}\right)-\left(\mathrm{A}_{4}\right)$ and (3.11), we get

$$
\begin{aligned}
\hat{v}(t)= & u(t ; f, g) \\
& +\int_{0}^{t}\left[W ( t - s ) \left[\int _ { 0 } ^ { s } \left\{k(s, \tau) G\left(\tau, \hat{v}_{\tau}\right)\right.\right.\right. \\
& \left.\left.\left.+H\left(s, \tau, \hat{v}_{\tau}\right)\right\} d \tau\right]+F\left(s, \hat{v}_{s}\right)\right] d s, \quad 0<t \leq t_{1}, \\
\hat{v}(\theta)= & g(\theta), \quad \theta \in[-h, 0] .
\end{aligned}
$$

This shows the local existence of a solution $v(t)=\left.\hat{v}(t)\right|_{\left[0, t_{1}\right]}$ of $(3.1)$ on $\left[0, t_{1}\right]$. Let $v_{1}$ and $v_{2}$ be the solution of (3.1) on $\left[0, t_{1}\right]$. Then it is easy to see, similarly to the above, that

$$
\left\|\hat{v}^{1}-\hat{v}^{2}\right\|_{C\left(\left[-h, t_{1}\right] ; H\right)} \leq L\left\|\hat{v}^{1}-\hat{v}^{2}\right\|_{C\left(\left[-h, t_{1}\right] ; H\right)},
$$

so that by $L<1, v_{1}(t)=v_{2}(t)$ on $\left[0, t_{1}\right]$. This proves the uniqueness.

Since $k(s, \tau) G\left(\tau, \hat{v}_{\tau}\right), H\left(s, \tau, \hat{v}^{n}\right), F\left(s, v_{s}\right) \in L^{2}\left(0, t_{1} ; H\right) \subset L^{2}\left(0, t_{1} ; V^{*}\right)$, by Theorem 2.1, we see that the solution $v(t)$ of (3.1) satisfies

$$
\begin{aligned}
\frac{d v(t)}{d t}= & A_{0} v(t)+A_{1} v(t-h)+\int_{-h}^{0} a(s) A_{2} v(t+s) d s \\
& +\int_{0}^{t}\left\{k(t, s) G\left(s, v_{s}\right)+H\left(t, s, v_{s}\right)\right\} d s \\
& +F\left(t, v_{t}\right)+f(t), \quad \text { a.e. } t \in\left[0, t_{1}\right], \\
v(\theta)= & g(\theta), \quad \theta \in[-h, 0],
\end{aligned}
$$

and $v \in L^{2}\left(0, t_{1} ; V\right) \cap W^{1,2}\left(0, t_{1} ; V^{*}\right)$. In this sense, we call this $v$ a mild solution of (1.5) on $\left[0, t_{1}\right]$. We give a norm estimation of the mild solution of (1.5) and establish the global existence of solutions with the aid of norm estimations. It is well known (cf. Lions and Magenes [3, Prop. 2.1, Thm. 3.1]) that the inclusion $L^{2}(0, T ; V) \cap$ $W^{1,2}\left(0, T ; V^{*}\right) \subset C([0, T] ; H)$ is continuous, that is, there exists a constant $c_{0}$ such that

$$
\|u\|_{C([0, T] ; H)} \leq c_{0}\left(\|u\|_{L^{2}(0, T ; V)}+\left\|\frac{d u}{d t}\right\|_{L^{2}\left(0, T ; V^{*}\right)}\right)
$$

for all $u \in L^{2}(0, T ; V) \cap W^{1,2}\left(0, T ; V^{*}\right)$.

LEMMA 3.1 [5]. Let $a(t), b(t)$, and $c(t)$ be real valued nonnegative continuous functions defined on $R^{+}$, for which the inequality

$$
c(t) \leq c_{0}+\int_{0}^{t} a(s) c(s) d s+\int_{0}^{t} a(s)\left[\int_{0}^{s} b(\tau) c(\tau) d \tau\right] d s
$$

holds for all $t \in R^{+}$, where $c_{0}$ is a nonnegative constant. Then

$$
c(t) \leq c_{0}\left(1+\int_{0}^{t} a(s) \exp \left[\int_{0}^{s}(a(\tau)+b(\tau)) d \tau\right] d s\right) \quad \text { for all } t \in R^{+} .
$$


THEOREM 3.2. Assume that the conditions in Theorem 3.1 hold. Then for any solution $v(t)=v(t ; f, g)$ of (3.1) on $[-h, T]$, we have the estimate

$$
\left\|v_{t}(\cdot ; f, g)\right\|_{C([0, T] ; H)} \leq c\left(|g(0)|+\|g\|_{L^{2}(-h, 0 ; V)}+\|f\|_{L^{2}\left(0, T ; V^{*}\right)}\right) e^{K t},
$$

where $c$ is a positive constant which does not depend on $v$.

Proof. From hypotheses $\left(\mathrm{A}_{1}\right)-\left(\mathrm{A}_{4}\right)$, we have

$$
\begin{aligned}
& |v(t+\theta ; f, g)| \\
& \quad \leq|u(t+\theta ; f, g)|+\mid \int_{0}^{t+\theta} W(t+\theta-s) \\
& \quad \times\left[\int_{0}^{s}\left\{k(s, \tau) G\left(\tau, v_{\tau}\right)+H\left(s, \tau, v_{\tau}\right)\right\} d \tau+F\left(s, v_{s}\right)\right] d s \mid \\
& \quad \leq|| u(\cdot ; f, g) \|_{C([0, T] ; H)} \\
& \quad+M \int_{0}^{t+\theta}\left[\int_{0}^{s}\left\{K\left|b_{1}(\tau)\right|\left\|v_{\tau}\right\|+\left|b_{2}(s, \tau)\right|\left\|v_{\tau}\right\|\right\} d \tau+\left|b_{3}(s)\right|\left\|v_{s}\right\|\right] d s .
\end{aligned}
$$

Hence, by (2.10) and (3.15),

$$
\begin{aligned}
\left\|v_{t}(\cdot ; f, g)\right\|= & \sup _{\theta \in[-h, 0]}|v(t+\theta ; f, g)| \\
\leq & K_{T} c_{0}\left(|g(0)|+\|g\|_{L^{2}(-h, 0 ; V)}+\|f\|_{L^{2}\left(0, T ; V^{*}\right)}\right) \\
& +\int_{0}^{t} c_{1}\left\|v_{s}(\cdot ; f, g)\right\| d s+\int_{0}^{t} \int_{0}^{s} c_{2}\left\|v_{\tau}(\cdot ; f, g)\right\| d \tau d s \\
\leq & c^{\prime}\left(|g(0)|+\|g\|_{L^{2}(-h, 0 ; V)}+\|f\|_{L^{2}\left(0, T ; V^{*}\right)}\right) \\
& +M\left(\int_{0}^{t}\left\|v_{s}(\cdot ; f, g)\right\| d s+\int_{0}^{t} \int_{0}^{s}\left\|v_{\tau}(\cdot ; f, g)\right\| d \tau d s\right) .
\end{aligned}
$$

By using Lemma 3.1, we get

$$
\begin{aligned}
\left\|v_{t}(\cdot ; f, g)\right\|_{C([0, T] ; H)} \leq & c\left(|g(0)|+\|g\|_{L^{2}(-h, 0 ; V)}+\|f\|_{L^{2}\left(0, T ; V^{*}\right)}\right) \\
& \times\left(1+M \int_{0}^{t} \exp \left(\int_{0}^{s}(M+1) d \tau\right) d s\right) \\
\leq & c^{\prime}\left(|g(0)|+\|g\|_{L^{2}(-h, 0 ; V)}+\|f\|_{L^{2}\left(0, T ; V^{*}\right)}\right) \\
& \times[1+M \exp \{(M+1) T\} t] \\
\leq & c\left(|g(0)|+\|g\|_{L^{2}(-h, 0 ; V)}+\|f\|_{L^{2}\left(0, T ; V^{*}\right)}\right) e^{K t}
\end{aligned}
$$

for some constants $c$ and $K$. This completes the proof.

By using Theorems 3.1, 3.2, we get the following theorem:

ThEOREM 3.3. Assume that the conditions in Theorem 3.1 hold. Then there exists a unique solution $v(t)$ on $[0, T]$ of (3.1) which satisfies the estimate

$$
\|v(\cdot ; f, g)\|_{C([0, T] ; H)} \leq c\left(|g(0)|+\|g\|_{L^{2}(-h, 0 ; V)}+\|f\|_{L^{2}\left(0, T ; V^{*}\right)}\right) e^{K T}
$$

for some constants $c$ and $K$. 
ACKNOWLEDGEMENT. This work was supported by KOSEF, 1996.

\section{REFERENCES}

[1] M. B. Dhakne and B. G. Pachpatte, On some abstract functional integrodifferential equations, Indian J. Pure Appl. Math. 22 (1991), no. 2, 109-134. MR 92e:47127. Zbl 728.45013.

[2] J. M. Jeong, S. Nakagiri, and H. Tanabe, Structural operators and semigroups associated with functional-differential equations in Hilbert spaces, Osaka J. Math. 30 (1993), no. 3, 365-395. MR 94i:34155. Zbl 818.47039.

[3] J. L. Lions and E. Magenes, Non-homogeneous boundary value problems and applications, Vol. I, Die Grundlehren der mathematischen Wissenschaften, vol. 181, SpringerVerlag, New York, Heidelberg, 1972, Translated from the French by P. Kenneth. MR 50 2670. Zbl 223.35039.

[4] S. Nakagiri, Existence, uniqueness and norm estimation for partial functional integrodifferential equations, Preprint.

[5] B. G. Pachpatte, A note on Gronwall-Bellman inequality, J. Math. Anal. Appl. 44 (1973), 758-762. MR 49 501. Zbl 274.45011.

[6] H. Tanabe, On fundamental solution of differential equation with time delay in Banach space, Proc. Japan Acad. Ser. A Math. Sci. 64 (1988), no. 5, 131-134. MR 89j:34090. Zbl 658.34056.

[7] _ Structural operators for linear delay-differential equations in Hilbert space, Proc. Japan Acad. Ser. A Math. Sci. 64 (1988), no. 8, 265-266. MR 89k:47061. Zbl 668.45013.

[8] _ Fundamental solutions for linear retarded functional-differential equations in Banach space, Funkcial. Ekvac. 35 (1992), no. 1, 149-177. MR 93f:34136. Zbl 771.34060 .

Park, Y. Lee, and J. LeE: Department of Mathematics, Pusan National University, PUSAN 609-735, KOREA 


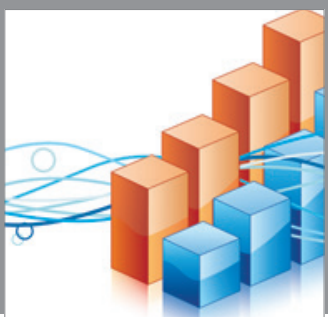

Advances in

Operations Research

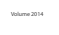

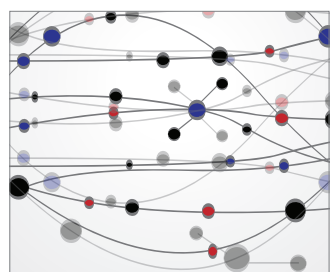

\section{The Scientific} World Journal
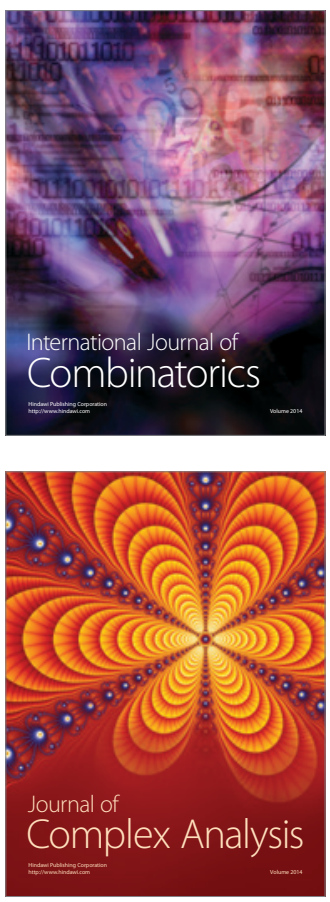

International Journal of

Mathematics and

Mathematical

Sciences
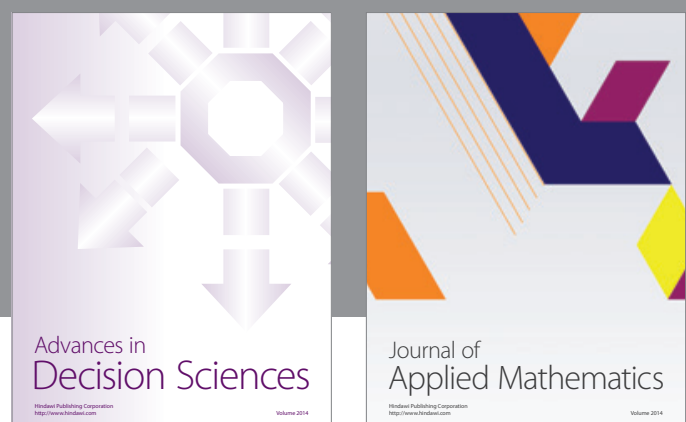

Journal of

Applied Mathematics
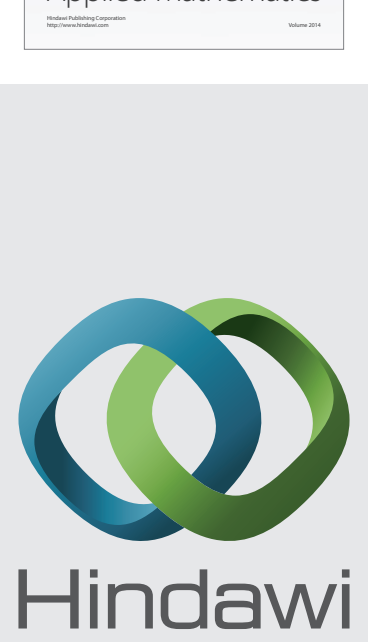

Submit your manuscripts at http://www.hindawi.com
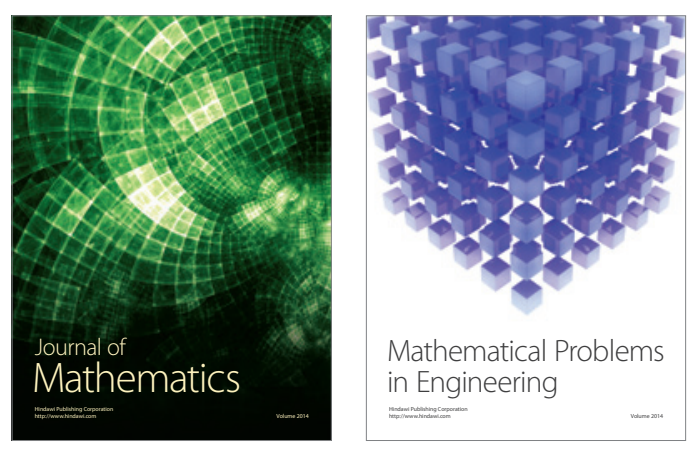

Mathematical Problems in Engineering
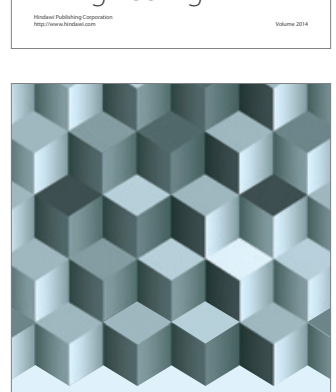

Journal of

Function Spaces
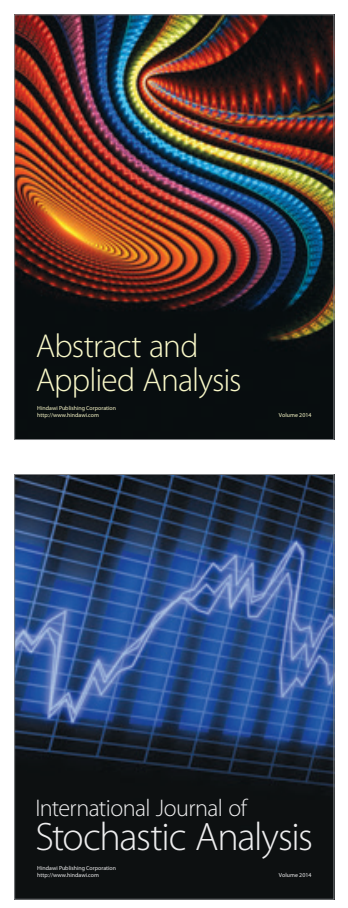

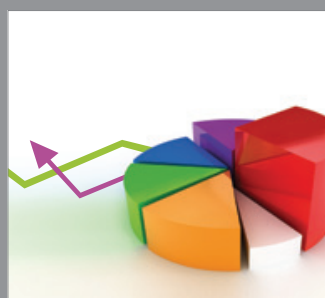

ournal of

Probability and Statistics

Promensencen
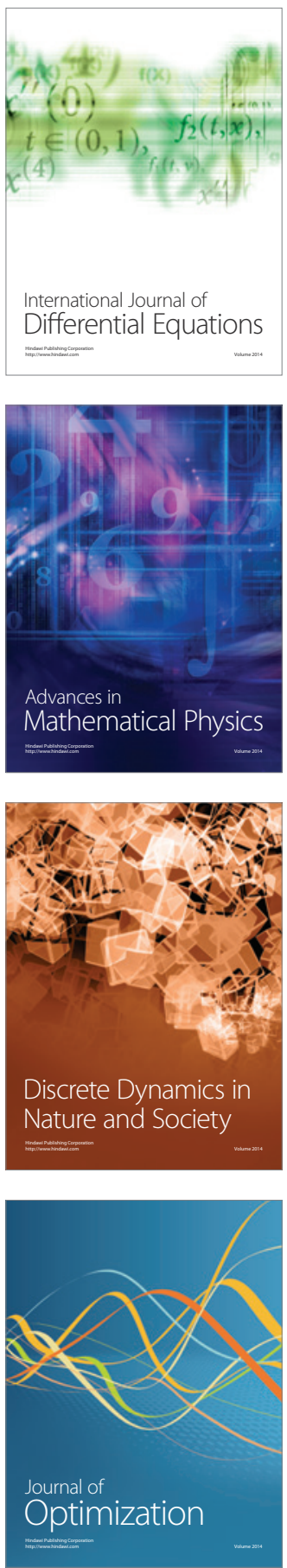